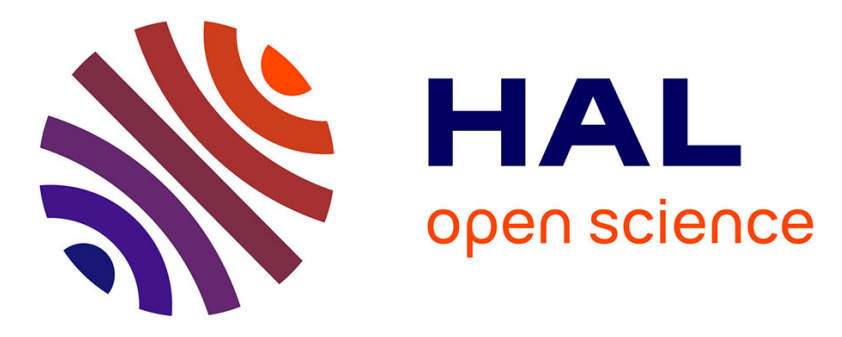

\title{
Characterization and modeling of magnetic domain wall dynamics using reconstituted hysteresis loops from Barkhausen noise
}

B Ducharne, Minh-Quyen Le, Gael Sebald, Pj Cottinet, D Guyomar, Y Hebrard

\section{- To cite this version:}

B Ducharne, Minh-Quyen Le, Gael Sebald, Pj Cottinet, D Guyomar, et al.. Characterization and modeling of magnetic domain wall dynamics using reconstituted hysteresis loops from Barkhausen noise. First world congress on condition monitoring, Jun 2017, London, United Kingdom. hal01570929

\author{
HAL Id: hal-01570929 \\ https://hal.science/hal-01570929
}

Submitted on 2 Aug 2017

HAL is a multi-disciplinary open access archive for the deposit and dissemination of scientific research documents, whether they are published or not. The documents may come from teaching and research institutions in France or abroad, or from public or private research centers.
L'archive ouverte pluridisciplinaire HAL, est destinée au dépôt et à la diffusion de documents scientifiques de niveau recherche, publiés ou non, émanant des établissements d'enseignement et de recherche français ou étrangers, des laboratoires publics ou privés. 


\title{
Characterization and modeling of magnetic domain wall dynamics using reconstituted hysteresis loops from Barkhausen noise.
}

\author{
B. Ducharne, MQ. Le, G. Sebald, PJ. Cottinet, D. Guyomar, Y. Hebrard* \\ Laboratoire de Génie Electrique et Ferroélectricité - INSA de Lyon \\ Bât. Gustave FERRIE, 8 rue de la physique, 69621 Villeurbanne cedex, France \\ *SKF-Aerospace, \\ 22 rue Brillat SAVARIN, 26958 VALENCE, France
}

Corresponding author: Ducharne B.

Benjamin.ducharne@insa-lyon.fr

Tél: +33 (0)4 $72438833 \quad$ Fax: +33 (0)4 72438874

\begin{abstract}
The characterization of the stress state in a material is important in the aeronautical and spatial sector, because the fatigue strength is intimately bounded to their internal stress distribution. The purpose of our work is to present and to develop a magnetic model of the Barkhausen noise to identify the stress profiles of the contact zones of the bearing raceways and rolling elements. By means of a post-processing technique, we succeeded in plotting magnetic Barkhausen noise energy hysteresis cycles MBNenergy $(\mathrm{H})$. These cycles were compared to the usual hysteresis cycles, displaying the evolution of the magnetic induction field $\mathrm{B}$ versus the magnetic excitation $\mathrm{H}$. The divergence between these comparisons as the excitation frequency was increased gave rise to the conclusion that there was a difference in the dynamics of the induction field and of the MBNenergy related to the domain wall movements. Indeed, for the MBNenergy hysteresis cycle, merely the domain wall movements were involved. On the other hand, for the usual $\mathrm{B}(\mathrm{H})$ cycle, two dynamic contributions were observed: domain wall movements and diffusion of the magnetic field excitation. From a simulation point of view, it was demonstrated that over a large frequency bandwidth a correct dynamic behavior of the domain wall movement MBNenergy $(\mathrm{H})$ cycle could be taken into account using firstorder derivation whereas fractional orders were required for the $\mathrm{B}(\mathrm{H})$ cycles. The present article also gives a detailed description of how to use the developed process to obtain the MBNenergy $(\mathrm{H})$ hysteresis cycle as well as its evolution as the frequency increases. Moreover, this article provides an interesting explanation of the separation of magnetic loss contributions through a magnetic sample: a wall movement contribution varying according to first-order dynamics and a diffusion contribution which in a lump model can be taken into account using fractional order dynamics.
\end{abstract}

\section{KEYWORDS}

Barkhausen noise, magnetic hysteresis loop, model, hysteresis, fractional derivative 


\section{Introduction}

Residual stresses are created by almost every manufacturing process. But despite their widespread occurrence, the fact that residual stress occur without any external loads makes them easy to overlook and ignore. This neglect can cause great design peril because residual stresses can have profound influences on material strength, dimensional stability and fatigue life. Sometimes alone and sometimes in combination with other factors, unaccounted for residual stresses have caused the failure aircraft and numerous smaller structures and devices often with substantial loss of life. Residual stresses can also be deliberately introduced to provide beneficial effects such as compressive state of bearing raceway.Since aircraft safety is an absolute requirement, the bearings are also required to have high reliability. SKF maintain high reliability by conducting full inspections in every step of bearing manufacturing process, such as procurement of material, forging, turning, heat treatment, machining and surface treatment, to verify manufacturing conformity including various certification contents mentioned in the previous section. In the event of any possible trouble such as damaged bearings, the ability to trace all manufacturing data of every process is also required.

With this tracing, a lot of empirical data are available and allow to identify grinding abuse. Residual stresses is more complex to identify. The Barkhausen signal here is dependent of many parameters. Full empirical approach for residual stresses using barkhausen noise necessitate a lot of expirement. Magnetic modelization is then needed to legitimate the signal measured.

The simulation of ferromagnetic devices involves the coupling of an accurate material constitutive law with Maxwell's equations [1]. Unfortunately, the implementation of a precise hysteresis model in magneto static or magneto dynamic simulations requires non-linear complex resolution techniques such as a fixed point including selfconsistency constraints [2]. To simplify such approach maintaining accurate simulation results, Pry and al. proposed a new simple domain configuration [3], others like Williams and al. focused on the velocity of propagation of a single domain boundary to understand and model correctly the whole hysteresis behavior [4]. In [5], the authors describe a good correlation between simulation/measurement by coupling the magnetic field diffusion equation to a frequency-dependent hysteresis model. In this representation, the authors claim that the diffusion equation was linked to the macroscopic eddy currents and that the frequency-dependent hysteresis model was derived from the movement of magnetic domain walls. Same approach is done by Maloberti and al. in [6]. For the hysteresis model used in [5], two contributions were required: a quasi-static, frequency-independent one, obtained using the usual quasistatic hysteresis model (Preisach model [7][8], Jiles-Atherton model [9][10], and so on [11]), and a dynamic contribution coming from the product of a constant $\rho$ related to the resistivity of the material and the first order derivation of the magnetic induction field $\mathrm{dB} / \mathrm{dt}$. The relatively complex coupled model that is obtained (diffusion/dynamic hysteresis) can be solved numerically.

Due to the non-linear behavior of the hysteresis, convergences of such simulations are however always hazardous and time-consuming. As detailed in [12] and [13], we have succeeded by replacing this complex coupled, space-dependent model (1-dimensional resolution of the diffusion equation) by a lump model with a unique dynamic term 
product of the same constant $\rho$ to the fractional derivation of the magnetic field. Good correlations between simulation/measurement were obtained here; both diffusion equation and hysteresis model contributions could be correctly taken into account by the fractional term [14]. This good correlation can be explained by the fact that the space decomposition of the 1-dimensional resolution has in the case of the diffusion of the magnetic field been replaced by the time decomposition of the fractional derivation term.

The Barkhausen noise BN effect corresponds to the noise in the magnetic output of a ferromagnetic as it is excited by an external, changing, applied, magnetizing force. BN was discovered by the German physicist H. Barkhausen in 1919; it originates from sudden changes in the size and orientation of ferromagnetic domains. BN occurs under the continuous process of magnetization or demagnetization and provides direct evidence of the existence of ferromagnetic domains which have previously only been postulated theoretically. It also gives interesting information about the movements of the domain walls and the energy related to this movement.

In [16] and [17], the authors defined a new parameter called the magnetic Barkhausen noise energy 'MBNenergy', obtained by integrating the square of the induced voltages with respect to time. In this article, a post-processing technique has been employed to obtain an hysteresis loop MBNenergy $(\mathrm{H})$ from the local magnetic Barkhausen noise measurement. By observing the evolution of this hysteresis loop as the frequency of the excitation field increased, we were able to make an interesting observation related to the dynamics of the wall movements. We could in particular confirm and illustrate the loss decomposition provided by Bertotti and al. in [18] and [19]. We could also provide precise frequency dependence of the hysteresis losses related to domain wall movements. The loss separation in this article is considered differently from Bertotti approach's. The quasi-static contribution is related to the hysteresis loop $\mathrm{B}(\mathrm{H})$ observed under excitation magnetic fields of frequencies lower than a few hertz. Indeed, for such weak frequency levels, whatever the excitation waveform, the hysteresis loop $B(H)$ is confined in a major hysteresis loop defined by extreme amplitude excitation. This quasistatic contribution is related to the hysteresis losses in Bertotti's approach. What we call the dynamic contribution is related to the difference of area appearing between hysteresis loop $\mathrm{B}(\mathrm{H})$ measured under quasi-static conditions and hysteresis loop $\mathrm{B}(\mathrm{H})$ resulting from similar waveform but for higher frequencies. This dynamic contribution will be the sum of the eddy currents and the excess losses in Bertotti's approach. In this work, we consider just two contributions for the loss separation. The quasi-static microscopic eddy current contribution and a dynamic component including both microscopic and macroscopic contributions. The microscopic behavior is related to the domain wall scale as well as the microscopic eddy currents are related to their movements. The macroscopic behavior is associated to the tested sample geometry scale and the macroscopic eddy currents related to induced eddy current appearing at that scale because of the magnetic field diffusion. We linked Barkhausen noise to microscopic eddy currents, and assume that even if the measure method used in this article is local and on the surface from a frequency dependence behavior, microscopic eddy currents will behave similarly whatever their position through the tested sample. The first part of this article describes a specific measuring set-up dedicated to Barkhausen characterization. It also presents the electronic analog circuit used to obtain the MBNenergy and the post-processing technique to get $\mathrm{MBNenergy}(\mathrm{H})$ hysteresis loops. The second part of this article exposes simulation models, both $\mathrm{MBNenergy}(\mathrm{H})$ 
and $\mathrm{B}(\mathrm{H})$ have been treated. Each contribution is described in detail and we particularly focus on the understanding of the frequency dependence of both hysteresis descriptions. Finally, a large number of measurement/simulation comparisons lead to the validation of each model, and to conclusions concerning loss separation and the evolution of their dynamics (wall movements, macroscopic eddy currents).

\section{Magnetic Barkhausen noise measuring setup}

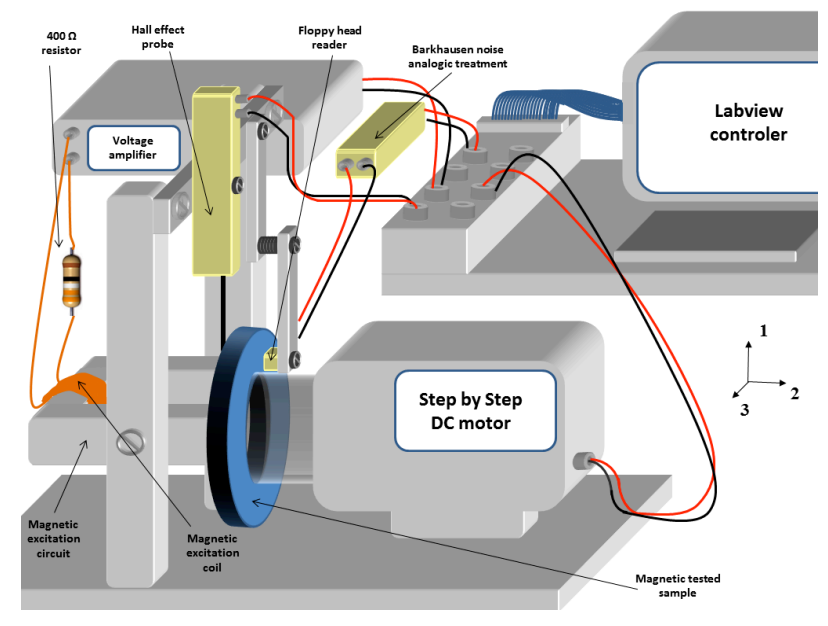

Figure 1: Magnetic Barkhausen noise measuring setup.

The measuring setup was developed in order to locally characterize a toroid magnetic circuit by measuring Barkhausen noise. The magnetic excitation field was provided by a coil of 4000 turns surrounding a high section $U$ magnetic circuit. The coils energy supply was obtained by a high-voltage KEPCO amplifier. A $400 \Omega$ power resistor was placed in series with the coil in order to obtain an undistorted waveform of the current even under high frequencies of the excitation. In this system, Barkhausen noise could be correctly detected for an excitation field frequency varying between a few $\mathrm{mHz}$ and approximately $50 \mathrm{~Hz}$. The surface excitation field $H$ was measured using a Hall effect sensor. This Hall probe was placed as close as possible to the Barkhausen sensor in order to correctly and locally defines the excitation field.

The left side of this sensor was in contact with the tested sample in order to ensure that the measured magnetic field corresponded to the surface field. A stepper $D C$ motor allowed circular displacements of the tested sample. This was particularly useful for the validation of the geographic reproducibility of our measurements. A read-write head from a $3 \frac{1}{2} 2$-inch unit floppy was used to detect the Barkhausen noise; the wall movements induced a weak voltage in the head coil which was transmitted to an electronic analog circuit. This circuit provided a first amplification of the Barkhausen signal, and once amplified this signal could be transmitted directly to the Labview controller or in the case of the $M B N_{\text {energy }}(H)$ observation, once again be analogically treated in a second electronic circuit.

Prior to this second electronic circuit, we use a high pass filter, model SR650, from Stanford Research Systems to ensure a correct $D C$ filtration. The cut-off frequency was set to $500 \mathrm{~Hz}$; we set an input gain of $40 \mathrm{~dB}$ and an output gain of $10 \mathrm{db}$ to amplify the Barkhausen noise amplitude. After this filtration, in a second electronic circuit, the 
square of the Barkhausen noise was first calculated using an AD633 analog multiplier, followed by a low noise operational amplifier OPA2604 in an integration configuration ensuring the integration of this signal. A small reed relay D31C2100 provided the reset function of the integrator as soon as the acquisition was completed. Figure 2 gives an illustration of the experimental setup we used for the acquisition of the $\operatorname{MBN}_{\text {energy }}(H)$ hysteresis cycles.

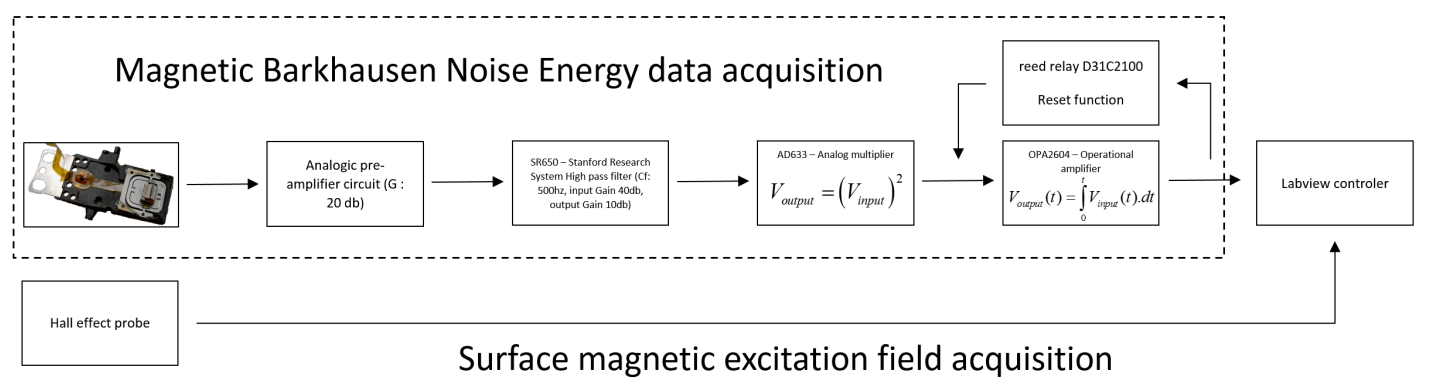

Figure 2: Scheme Block picture of the experimental setup used for the acquisition of the $M B N_{\text {energy }}(H)$ hysteresis loop.

\section{Experimental results and discussion}

In order to validate all the statements put forward in this article, several comparisons between simulation/measurement were performed. The first results were related to comparisons between simulation/ measurement for the $\mathrm{B}(\mathrm{H})$ hysteresis cycle under high-amplitude magnetic excitation fields. The good results obtained by our simulation made it possible to validate the fractional consideration of the dynamic losses. It confirmed - as already published in [23] - that both the microscopic and macroscopic contributions of the eddy current dynamics can be correctly taken into account by fractional derivation. In this study, M50NIL steel provided by SKF is chosen to be implemented on the experimental test bench. The sample is steel massive, torus form similarly to a bearing shape, with $70 \mathrm{~mm}$ of external diameter and $10 \mathrm{~mm}$ of thickness. This material benefits excellent thermal stability with significantly increased toughness as well as high resistance to fractures lead to small changes in bearing ring dimensions which may add to subsequent distortions caused by heat-treatment [24][25]. Such properties are the determining factors that make M50NIL as one of the most appropriate material choice for bearing of the aero-engine." M50 indicated that the tested steel comprised an addition of molybdenum as one of the primary alloying elements. The ' $\mathrm{Ni}$ ' in the name stands for nickel while ' $\mathrm{L}$ ' refers to low carbon. Table 1 gives the approximate chemical compositions in weight $\mathrm{t} \%$.

\begin{tabular}{l|l|l|l|l|l|l|l}
\multicolumn{1}{l|}{} & $\mathrm{C}$ & $\mathrm{Si}$ & $\mathrm{Cr}$ & $\mathrm{Mo}$ & $\mathrm{V}$ & $\mathrm{Ni}$ & $\mathrm{Mn}$ \\
\hline M50NIL & 0.13 & 0.18 & 4.1 & 4.25 & 1.2 & 3.5 & 0.2
\end{tabular}

Table 1: approximate chemical compositions of M50NIL in weight $\mathrm{t} \%$. 

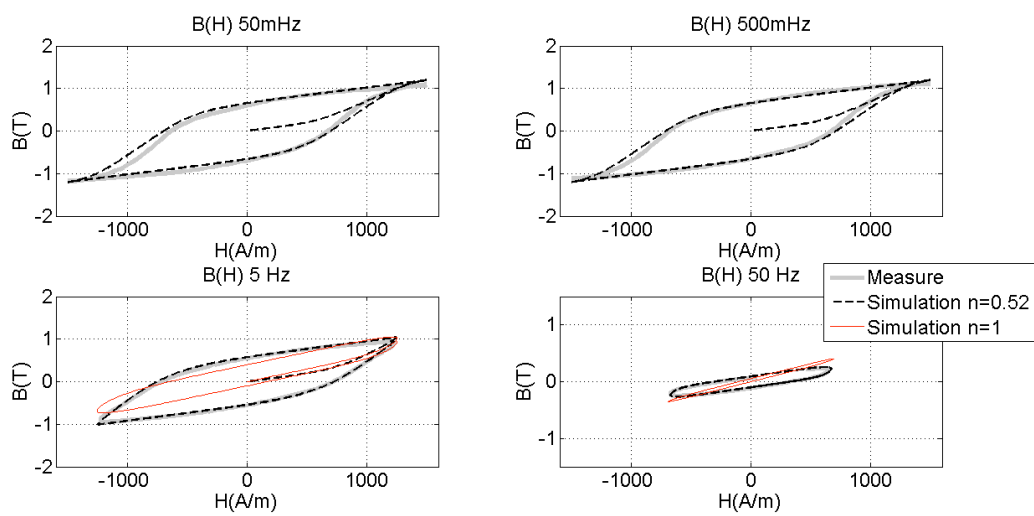

Figure 4: Comparison between $B(H)$ simulation/measurement for frequencies varying from $50 \mathrm{mHz}$ to $50 \mathrm{~Hz}$.

As illustrated in Figure 4, good comparisons were obtained between measurements and simulations when we employed fractional order dynamic consideration. Figure 5 confirms this too, illustrating in particular the overestimation of the first-order dynamic consideration as soon as we exceeded an excitation of a few hertz.

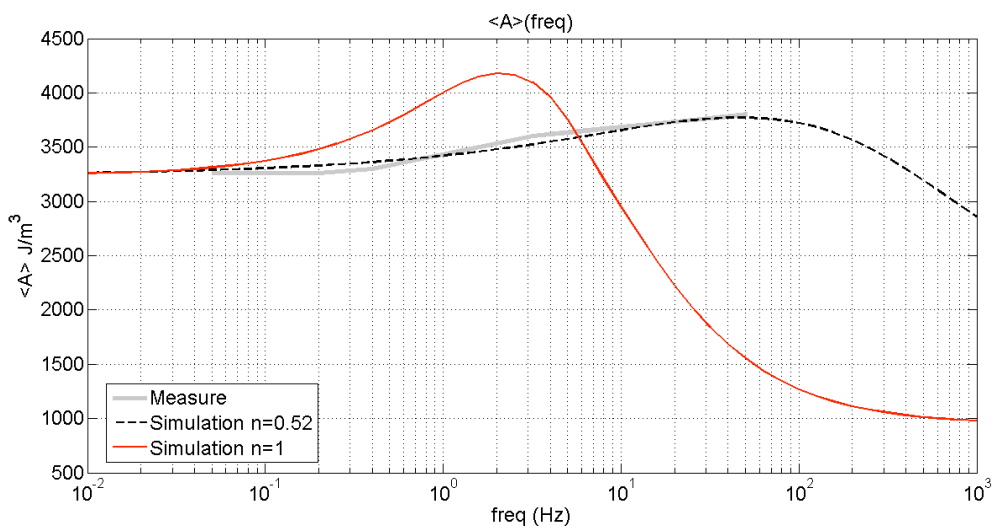

Figure 5: Comparison between simulation/measurement for curves of hysteresis loop area versus frequency $\langle A>$ (freq) $(<\mathrm{A}>$ is related to the hysteresis loop area).

Let us now verify the Barkhausen results. The objective here was to confirm that the wall movement dynamics were first order. Almost three decades of frequency were tested, from $100 \mathrm{mHz}$ to $50 \mathrm{~Hz}$. These values were chosen as extremum frequencies since our measuring setup provides particularly well-defined experimental results in this frequency bandwidth, but unfortunately this is no longer the case as soon as we explore higher frequencies. The same toroid sample was tested. Measurements were performed using the measuring setup described in the second part of this article.

As illustrated in Figure 6, good comparisons were obtained between measurements and simulations when we employed a first-order dynamic consideration. Figure 7 confirmed this too, illustrating in particular, and contrary to the $B(H)$ behavior, the underestimation of the fractional dynamic consideration as soon as we exceeded an excitation of a few hertz. 

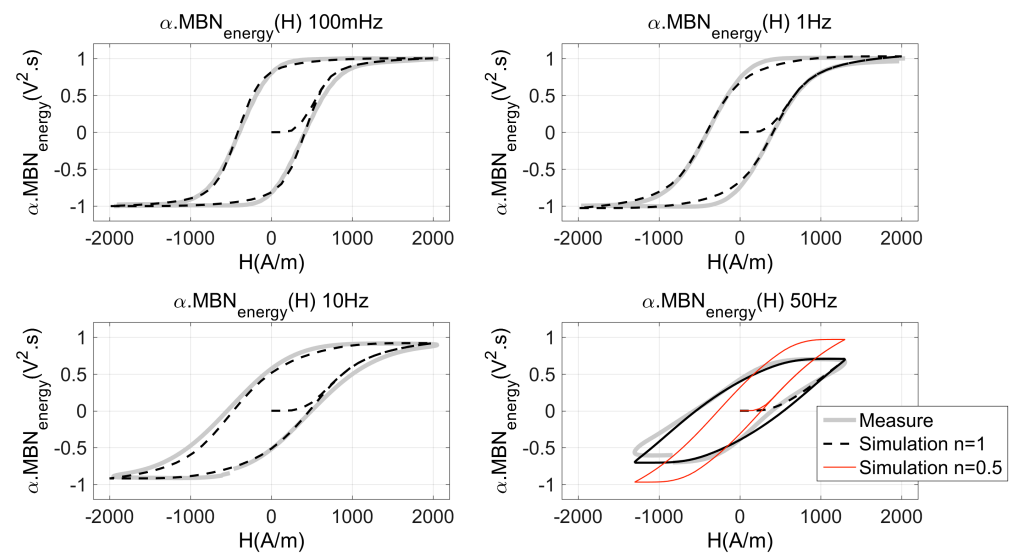

Figure 6: Comparison between $\alpha M B N_{\text {energy }}(H)$ simulation/measurement, for frequencies varying from $50 \mathrm{mHz}$ to $50 \mathrm{~Hz}$.

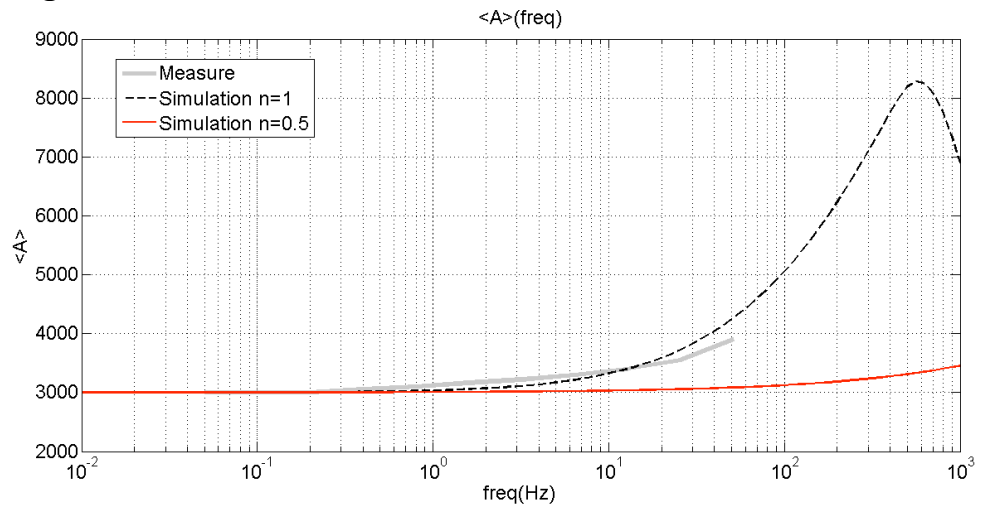

Figure 7: Comparison between simulation/measurement for curves of hysteresis loop area $\alpha M B N_{\text {energy }}(H)$ versus frequency $<A>($ freq $)$.

Finally, a discussion can be given to illustrate the differences between our approach and Bertotti's loss decomposition. For a given Induction B(t), Bertotti's formulation means to separate the total excitation field $\mathrm{H}$ in three contributions:

$H_{\text {total }}(t)=H_{\text {static }}(B(t))+H_{\text {eddycurrents }}+H_{\text {excel }}$

Where $H_{\text {static }}(B(t))$ is the quasi-static contribution, it is frequency independent. In the Bertotti's losses distribution, it is related to the hysteresis losses. $H_{\text {eddycurrents }}$ is linked to macroscopic eddy currents. In the Bertotti's formulation this excitation field contribution is considered by the product of a constant $\gamma$ to the time derivation of the magnetic induction field. $\gamma$ depends on the conductivity $\sigma$ and on the geometry of the tested sample $\left(d^{2} / 12\right.$, in the case of magnetic sheet samples where $d$ is the thickness and 1 the length and assuming $1>>d$ ).

$H_{\text {eddycurrents }}=\gamma \cdot \frac{d B}{d t} \quad \gamma=\frac{\sigma \cdot d^{2}}{12}$

Finally, $H_{\text {excel }}$ is linked to the dynamic of the magnetic domain walls (microscopic eddy currents) and in the Bertotti's formulation it is equal to the product of a constant $\alpha$, the sign of the time derivation of the magnetic induction and the square root of the absolute value of the time derivation of the magnetic induction.

$H_{\text {excel }}=\alpha . \delta .\left|\frac{d B}{d t}\right|^{1 / 2} \quad \alpha=\sqrt{\sigma G V_{0} S} \quad \delta=\operatorname{sign}\left(\frac{d B}{d t}\right)$ 
Where $\mathrm{S}$ is the section area, $\mathrm{V}_{0}$ is a phenomenological parameter characterizing a given material. $\mathrm{G}$ is a constant:

$G=\frac{4}{\pi^{3}} \sum_{\text {odd }} \frac{1}{n^{3}} \quad 0.1356$

In our formulation, for a given Induction field $\mathrm{B}(\mathrm{t})$, our formulation also means to separate the total excitation field $\mathrm{H}$ but just in two contributions (last version of eq. 4). Equation 1 gives the quasi-static contribution (similar at Bertotti's quasi-static contribution) and eddy current contribution and excess losses contribution are considered in a single term, product of a constant $\rho$ to the fractional time derivation of the magnetic induction. $\alpha$ the fractional order is a material parameter, in the case of the M50NIL the best results on a large frequency bandwidth are obtained with a $\alpha$ value close to 0.52 . From a frequency point of view, if we assume both sinus magnetic excitation and induction fields, eddy currents contribution is in $f^{0.5}$ and excess losses in $f^{1}$ for the Bertotti's formulation. In our fractional formation, the frequency dependence of our fractional term which include both macroscopic and microscopic contributions is in $f^{0.52}$ for the M50NIL. The M50NIL as a high electric conductivity ( $\left.>110^{7}{\mathrm{~S} . \mathrm{m}^{-1}}^{-1}\right)$ which means that the macroscopic contribution is quickly preponderant as soon as we increase the frequency, this justifies a fractional order (0.52) close to 0.5 .

\begin{tabular}{l|l|l|l|} 
& Static contribution & eddy currents & excess losses \\
\hline $\begin{array}{l}\text { Bertotti's } \\
\text { formulation }\end{array}$ & frequency independent & $f^{0.5}$ & $f^{1}$ \\
\hline $\begin{array}{l}\text { fractional } \\
\text { formulation }\end{array}$ & frequency independent & $f^{0.52}$ & \\
\hline
\end{tabular}

Table 2. Synthetic table for the frequency dependence of the excitation field contributions, comparison Bertotti's formulation / fractional model.

From a magnetic loss decomposition point a view, still considering harmonic magnetic and induction field and using hysteresis area $<A>$, i.e. energy lost during one period of excitation field per volume quantity, as energy unit:

$<A>=\int_{0}^{T} H \cdot d B$

The frequency dependence becomes:

\begin{tabular}{l|l|l|l|} 
& Static contribution & eddy currents & excess losses \\
\hline $\begin{array}{l}\text { Bertotti's } \\
\text { formulation }\end{array}$ & frequency independent & $f^{1.5}$ & $f^{2}$ \\
\hline $\begin{array}{l}\text { fractional } \\
\text { formulation }\end{array}$ & frequency independent & $f^{1.52}$ & \\
\hline
\end{tabular}

Table 3. Synthetic table for the frequency dependence of the magnetic losses contributions, comparison Bertotti's formulation / fractional model.

\section{Conclusion}

Thanks to a post-processing technique, we were able to successfully plot magnetic Barkhausen noise energy hysteresis cycles, $M B N_{\text {energy }}(H)$. These cycles were then compared to usual hysteresis cycles, i.e., the evolution of the magnetic induction field $B$ 
versus the magnetic excitation $H$. The observed divergence between these comparisons as the excitation frequency increased led to the conclusion that the dynamics of the induction field and of the $M B N_{\text {energy }}$ related to the domain wall movements were different. Indeed, for the $M B N_{\text {energy }}$ hysteresis cycle, only the domain wall movements were involved whereas for the usual $B(H)$ cycle two dynamic contributions were observed: the domain wall movements and the diffusion of the magnetic field excitation. It was also demonstrated that over a large frequency bandwidth a correct dynamic behavior of the domain wall movement for $M B N_{\text {energy }}(H)$ cycles could be taken into account using first-order derivation while fractional orders were required for the $B(H)$ cycles. All these observation led to an interesting conclusion for the separation of the magnetic loss contributions through a magnetic sample: a wall movement contribution varying according to first-order dynamics and a diffusion contribution which in a lump model can be taken into account using fractional order dynamics.

\section{References}

[1] J. Saitz, Newton-Raphson Method and Fixed-Point Technique in Finite Element Computation of magnetic Fields Problems in Media with Hysteresis, IEEE Trans. on Mag. 35 (1999) 1398-1401.

[2] F Henrotte, Modelling ferromagnetic materials in 2D finite element problems using Preisach's model, IEEE Trans. on Mag. 28 (1992) 2614-2616.

[3] R.H.Pry, C.P. Bean, Calculation of the energy loss in magnetic sheet materials using a domain mode, J. of App. Phys. 29 (1958) 532-533.

[4] H.J. Williams, W. Shockley, C. Kittel, Studies of the propagation velocity of a ferromagnetic domain boundary, Phys. Rev. 80 (1950) 1090-1094.

[5] M.A. Raulet, B. Ducharne, JP. Masson, G. Bayada, The magnetic field diffusion equation including dynamic hysteresis: A linear formulation of the problem, IEEE Trans. on Mag. 40 (2004) 872-875.

[6] O. Maloberti, A. Kedous-Lebouc, O. Geoffroy, G. Meunier, V. Mazauric, Field diffusion-like representation and experimental identification of a dynamic magnetization property, J. of Mag. and Mag. Mat. 304 (2006) 507-509.

[7] F. Preisach, Uber die Magnetische Nachwirkung, Z. Phys. 94 (1935) 277-302.

[8] G. Biorci, D. Pescetti, Analytical Theory of the behaviour of ferromagnetic materials; Il Nuovo Cimento, 7 (1958) 829-843.

[9] S.E. Zirka, Y.I. Moroz, P. Marketos, A.J. Moses, D.C. Jiles, Measurement and modeling of B-H loops and losses of high silicon nonoriented steels, IEEE Trans. on Mag. 42 (2006) 3177-3179.

[10] D.C.Jiles, D.L. Atherton, theory of ferromagnetic hysteresis, J. of App. Phys. 55 (1984) 2115-2120.

[11] A. Nourdine, A. Kedous-Lebouc, G. Meunier, T. Chevalier, A chemical reaction hysteresis model for magnetic materials, IEEE Trans. on Mag. 36 (2000) 1230-1233.

[12] B.Ducharne, D. Guyomar, G.Sebald, Low frequency modelling of hysteresis behavior and dielectric permittivity in ferroelectric ceramics under electric field, J. of Phys. D - App. Phys. 40 (2007) 551-555.

[13] D.Guyomar, B.Ducharne, G.Sebald, Dynamical hysteresis model of ferroelectric ceramics under electric field using fractional derivatives, J. of Phys. D - App. Phys. 40 (2007). 
[14] D.Guyomar, B.Ducharne, G.Sebald, The use of fractional derivation in modeling ferroelectric dynamic hysteresis behavior over large frequency bandwidth, J. Appl. Phys. 107 (2010).

[15] B.Zhang, B. Ducharne, D. Guyomar, G. Sebald, Energy harvesting based on piezoelectric Ericsson cycles in a piezoceramic material, Eur. Phys. J. - Spec. Top. 222 (2013) 1733-1743.

[16] T.W. Krause, J.A. Szpunar, D.L. Atherton, Anisotropic flux density dependence of Magnetic Barkhausen Noise in oriented 3\% Si-Fe steel Laminates, IEEE Trans. on Mag. 39 (2003) 562-566.

[17] M. Birsan, J.A. Szpunar, T.W. Krause, D.L. Atherton, Magnetic Barkhausen noise study of domain wall dynamics in grain oriented 3\% Si-Fe, IEEE Trans. on Mag. 32 (1996) 527-534.

[18] G. Bertotti, General properties of power losses in soft ferromagnetic materials, IEEE Trans. on Mag. 24 (1988) 621-630.

[19] G. Bertotti, Hysteresis in magnetism for physicists, Academic Press, San Diego, 1998.

[20] M.A. Raulet, J.P. Masson, Gaude D., Introduction of a dynamical model of material in the magnetic field diffusion equation, Eur. Phys. J. - App. Phys. 1 (1998) 7376.

[21] F. Marthouret, J.P. Masson, H. Fraisse, Modeling of a non-linear conductive magnetic circuit, IEEE Int. Mag. Conf. (INTERMAG 95), San Antonio Texas, 1995.

[22] M.A. Raulet, J.P. Masson, D. Gaude, Presentation and experimental validation of a behavioral model of dynamic properties for magnetic materials, J. Phys. IV 8 (1998) 619-622.

[23] B.Ducharne, G. Sebald, D. Guyomar, G. Litak, Dynamics of magnetic field penetration into soft ferromagnets, J. Appl. Phys. 117 (2015).

[24] H.K.D.H. Bhadeshia, "Steels for bearings," Prog. Mater. Sci. 57 (2012) 268-435.

[25] H.J. Böhmer, F. J. Ebert, W. Trojahn. "M50NiL bearing material - heat treatment, material properties and performance in comparison with M50 and RBD”, Lub.Eng., 48 (1992) 28-35. 